\title{
Ataxin-2, a new target in complex genetic diseases
}

\author{
Sergio Alberto Ramírez-García, ${ }^{1}$ José Sánchez-Corona, ${ }^{2}$ Diego Ortega-Pacheco, ${ }^{3}$ \\ Eric Ramírez-Bohórquez ${ }^{3}$ and Diana García-Cruz ${ }^{4}$ \\ ${ }^{1}$ Universidad de la Sierra Sur, Institute of Public Health Research, Oaxaca; ${ }^{2}$ Instituto Mexicano del Seguro Social, Center for Biomedical Research \\ of the West, Molecular Medicine Division, Jalisco; ${ }^{3}$ Universidad de la Sierra Sur, Masters' Degree in Public Health Program, Oaxaca; ${ }^{4}$ Universidad \\ de Guadalajara, University Center of Health Sciences, Institute of Human Genetics "Dr. Enrique Corona Rivera", Jalisco, Mexico
}

\begin{abstract}
The ataxin 2 gene is a target in the pathogenesis of complex diseases, including cardiovascular risk factors and neurodegenerative diseases. ATXN2 gen has VNTR in exon 1, whose expansion exceeding 30 repetitions leads to the development of spinocerebellar ataxia type 2; lower-range repetitions are associated with type 2 diabetes or amyotrophic lateral sclerosis. This locus is also linked with metabolic and inflammatory phenotypes. In conclusion, this gene can be used as a clinical marker of metabolic and neurological phenotypes, which is related to its pleiotropic effect.
\end{abstract}

KEY WORDS: Ataxin 2. Glaucoma. Inflammation. Microcirculation. Parkinsonism.

Complex diseases such as type-2 diabetes mellitus, dyslipidemia, obesity, hypertension, coronary heart disease, microangiopathic problems and dementia, among others, do develop by the additive effect of environmental factors, such as nutritional status and genetic factors. The common element is a simultaneous involvement of several organs or multiple organ resistance. In this sense, the mapping of associated genes requires the consideration of pleiotropic genes, which have simultaneous effects on various organs and systems; one of them is the gene that encodes ataxin-2 (ATXN2). ${ }^{1-2}$

Ataxin-2 is an RNA-binding protein that interacts with PABPC1 and is located in ribosomes and the rough endoplasmic reticulum. In situations of cellular stress, both proteins are relocated in granules, where mRNAs are protected from translation and degradation. It also modifies the abundance of basal translation factors, global translation and is a modulator of ribosomal biogenesis through the regulation of transcription factors such as Nop10, Rps10, rps18, RPL14, Rpl18 and Gnb2l1; as well as translation initiation through the Eif2s2, ElF3S6, elF4B, Pabpc1, Srp14, bR1, SEC61B factors and REr translocase. ${ }^{3}$
The gene for ataxin-2 is regulated by the $A 2 B P 1$ gene, which encodes for one of its binding proteins, whose deficiency in mouse embryonic hypothalamic cells causes a decrease in the atxn2, Insr and Mc4r genes, which is associated with the development of obesity. ${ }^{4}$ This study suggests that ATXN2 is involved in energy balance at the hypothalamic level, which is supported by two other murine models: one that shows ataxin-2 deficiency and loss of function, which translates into marked hyperphagia; $;^{5-7}$ the second one is a knock-out model. The clinical phenotype of these mice is severe obesity, fatty liver, cholesterol metabolism abnormalities and insulin receptor underexpression in the liver and brain. ${ }^{8}$ The mechanism by means of which these alterations occur is still under study.

In animal models, atxn2 overexpression has been reported to result in neurotoxicity, in conjunction with the atxn1, atxn3, tdp-43 and mapt proteins (also responsible for neurodegeneration in spinocerebellar ataxias [SCA], amyotrophic lateral sclerosis [ALS], frontotemporal dementias and progressive supranuclear palsy in humans). Neuronal atrophy occurs due to ATXN2 gain of function, which is related to an increase exon 1-encoded polyglutamines tract, which is
Correspondence:

Diana García-Cruz

E-mail: dianagarcr@hotmail.com
Date of reception: 20-05-2017

Date of acceptance: 09-01-2018

DOI: 10.24875/GMM.M19000232
Gac Med Mex. 2019;155:56-59

Contents available at PubMed www.gacetamedicademexico.com 


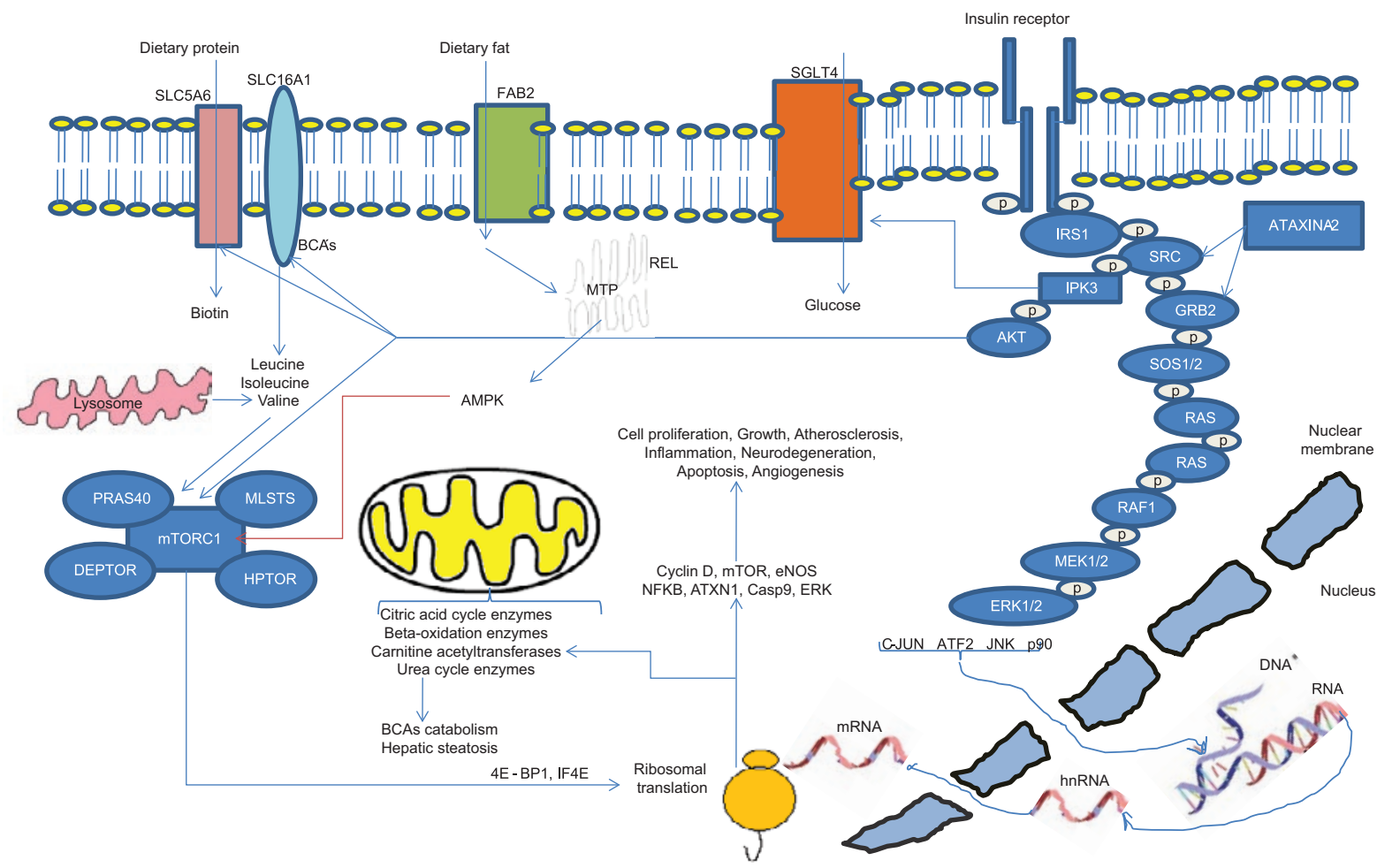

Figure 1. Ataxin-2 genomics, proteomics and metabolomics.

explained by the fact that ataxin-2 interacts with the RTK endocytosis modulator complex $\mathrm{SH} 3$ domain. ${ }^{9}$ In turn, ATXN2 deficiency mitigates neurotoxicity and leads to neuroblastoma cell lines apoptosis, which is associated with Grb2 and SRC decreased levels (Figure 1) and has been demonstrated in mutant mice and in human skin fibroblast cultures.

The above observations suggest that ATXN2 is a modulator of neuronal atrophy via the STK signaling pathway; through AKT, it regulates ataxin-1, which is related to neurodegeneration in spinocerebellar ataxia type 1 (Figure 1). ${ }^{9}$

Molecular genetics studies in humans show the direct relationship of the ATXN2 gene with the development of neurodegenerative disorders such as spinocerebellar ataxia type 2 (SCA2), ALS or post-polio syndrome, by expansion of the unstable CAG sequence repetition in exon 1 of the gene, which encodes a polyglutamine, although CAA interruptions in CAG triplets expansion also contribute to the clinical phenotype of these disorders. Linkage of the ATXN2 locus, where VNTR is located, predisposes to the development of type 1 diabetes mellitus and celiac disease, which has also been associated with SH2B3 deregulation. ${ }^{10}$ In the English population, CAG expansion in the pre-mutation range (more than 22 repeated alleles) and homozygous $C$ status for the rs695872 SNP that flanks VNTR, have been observed to be associated with the development of severe obesity (analogous to mutations in the genes for leptin, leptin receptor and melanocortin type 4 receptor). ${ }^{5}$ Recently, the association of pure type 2 diabetes mellitus with CAG repetitions in the pre-mutation range was shown in a case-control study. ${ }^{11}$

Studies exhaustively searching for the human genome have revealed the association of the ATXN2 gene with metabolic syndrome metabolic and inflammatory parameters, ${ }^{12,13}$ as well as with hematological parameters (hemoglobin levels, erythrocyte, leukocyte and platelet counts and volume) in the development of coronary artery disease and myocardial infarction. ${ }^{14}$ In Caucasian populations, the locus is associated with retinal vascular/venular caliber, coronary heart disease and hypertension. ${ }^{15}$ An epidemiological study in European population showed a correlation with erythrocyte traits, hemoglobin mean concentration, hemoglobin concentration, hematocrit, mean corpuscular volume, corpuscular hemoglobin concentration and erythrocyte count. ${ }^{16}$

Previous research suggests that cytoplasmic ataxin-2 plays a key role in metabolic homeostasis in different tissues, since it is a nutritional and metabolic modulator 


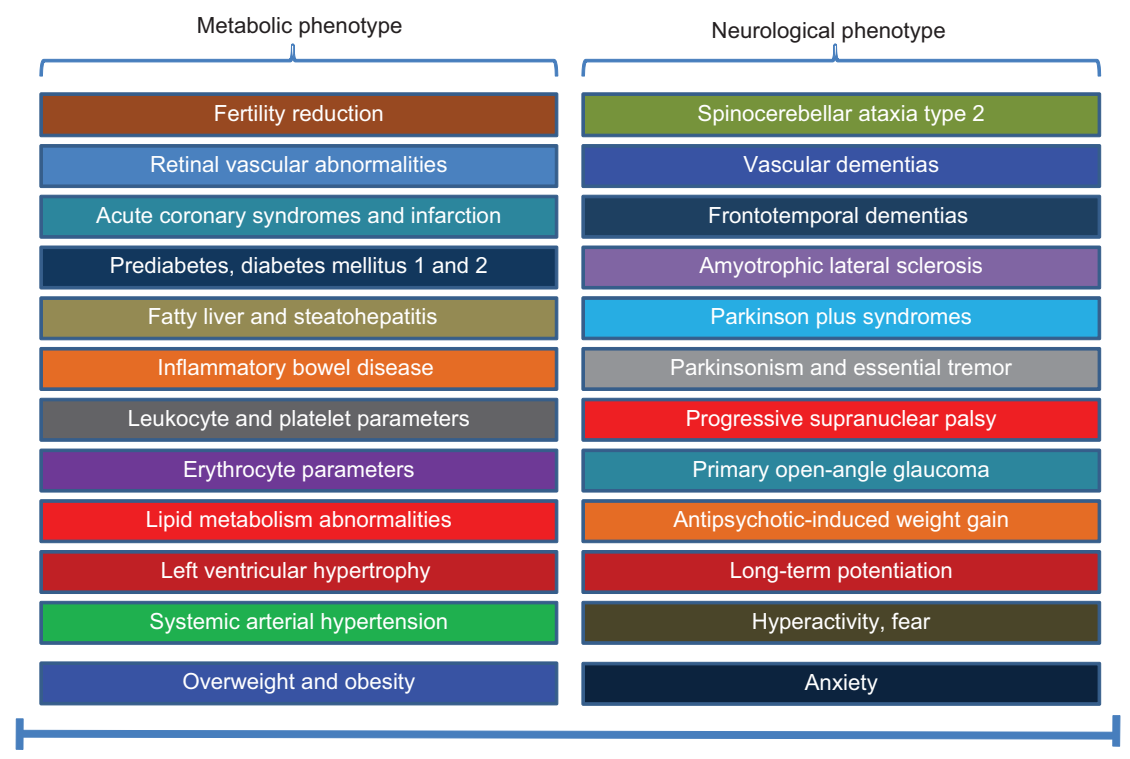

Figure 2. Ataxin-2 clinical expression.

that is sensitive to cellular stress. In embryonic fibroblasts, it leads to increased phosphorylation of translation factor 4E-BP1, as well as of ribosomal protein S6, via PI3K-mTOR. ${ }^{7}$ On the other hand, it alters RNA processing and trophic receptors internalization. ${ }^{10}$

These observations are supported by the results of an ataxin-2 knock-out model, where the liver of mice shows a significant decrease in proteins related to fat oxidation, branched-chain amino acids degradation, ketogenesis and citric acid cycle, such as ACADS, ALDH6A1, ALDH7A1, IVD, MCCC2, PCCA and OTC. ${ }^{17}$ Therefore, in humans, the ATXN2 gene, like its homologue in mice, participates in abnormalities related to insulin resistance, obesity, lipid metabolism alterations, hypertension, glucose homeostasis disorders and severe polyphagia, among other inflammatory and vascular metabolic traits. ${ }^{18}$

ATXN2 gene deficiency clinical-nosologic spectrum is also related to behavioral problems and obesity, as it has been demonstrated in humans and mice. Knockout mice develop reduced fertility, hyperactivity, decreased innate fear, impaired long-term enhancement of the amygdala; amygdala altered plasticity is associated with a reduction of key and context-conditioned fear. This animal model may be useful for the study of anxiety disorders and should encourage the investigation of anxiety in patients with spinocerebellar ataxia type 2 (SCA2). ${ }^{19}$ In humans, linkage studies reveal that ataxin gene 12q24.12 locus is associated with weight gain by the consumption of antipsychotics. ${ }^{5}$

Indeed, ataxin-2 clinical phenotypic spectrum is broad (Figure 2); its variation and genetic expression are related to clinical development of two phenotypes: metabolic-inflammatory-vascular and neurological-behavioral, which reflects the pleiotropic effect. Owing to all ataxin-2 physiological implications, its gene is postulated as a candidate in epidemiological studies of genetics, epigenetics, silencing, interference RNA, gene therapy and antisense therapy for nutrition-related complex diseases, such as metabolic syndrome and neurodegenerative and human behavior disorders, which constitute public health problems in Mexico.

In vitro experimental studies with ATXN2-targeted antisense therapy appear promising in SCA2 and ALS. ${ }^{20-23}$ Recently, interactomics between ataxin-2 and EIF4G1/PARK18 has been targeted in Parkinson's disease. ${ }^{23}$ One of the genes responsible for ALS, GSTO2, has been associated with SCA2 age of onset, particularly the rs2297235 heterozygous state. ${ }^{24}$ In a similar sense, the ATXN2 locus is a modulator of type 3 spinocerebellar ataxia age of onset. ${ }^{25}$ These results reveal that the complex phenotype of neurodegenerative diseases may be influenced by gene and protein interactomics.

In studies with stem cells, several isotypes of cell lines for spinocerebellar ataxia type 2 have been generated, including IPSC H196, 221, 227 and 266, although they continue under research as a therapeutic option. ${ }^{24-29}$ Through large-scale genome search or comprehensive genomic search with SNP analysis, the relationship with cardiac structure and function has been explored, where ATXN2 rs10774625 polymorphism is associated with left ventricle size, while 
rs7137828 is associated with the development of glaucoma, with an odds ratio of 1.17 for the T allele of this genetic variant. ${ }^{22,29}$ Recent advances in the study of the ATXN2 gene reveal that its deficiency in the knock-out model causes a deregulation of the calcium metabolism, which will have to be explored in future studies in patients with spinocerebellar ataxia type $2 .^{30}$

In conclusion, the ATXN2 gene can be used as a genetic marker in clinical practice for prognosis or diagnosis, especially for metabolic, vascular, inflammatory and neurological diseases, regardless of the etiology.

\section{References}

1. Nkiliza A, Chartier-Harlin MC. ATXN2 a culprit with multiple facets. Oncotarget. 2017;8:34028-34029.

2. Lastres-Becker I, Rüb U, Auburger G. Spinocerebellar ataxia 2 (SCA2) Cerebellum. 2008:7:115-124.

3. Fittschen M, Lastres-Becker I, Halbach MV, Damrath E, Gispert S, Azizov M, et al. Genetic ablation of ataxin-2 increases several global translation factors in their transcript abundance but decreases translation rate. Neurogenetics. 2015;16:181-192.

4. Ma L, Hanson RL, Traurig MT, Muller YL, Kaur BP, Perez JM, et al. Evaluation of A2BP1 as an obesity gene. Diabetes. 2010;59:2837-2845.

5. Figueroa KP, Faroogi S, Harrup K, Frank J, O'Rahilly S, Pulst SM. Genetic variance in the spinocerebellar ataxia type 2 (ATXN2) gene in children with severe early onset obesity. PLoS One. 2009;4:e8280.

6. Kiehl TR, Nechiporuk A, Figueroa KP, Keating MT, Huynh DP, Pulst SM. Generation and characterization of Sca2 (ataxin-2) knockout mice. Biochem Biophys Res Commun. 2006;339:17-24.

7. Lastres-Becker I, Nonis D, Eich F, Klinkenberg M, Gorospe M, Kötter P, et al. Mammalian ataxin-2 modulates translation control at the pre-initiation complex via PI3K/mTOR and is induced by starvation. Biochim Biophys Acta. 2016;1862:1558-1569.

8. Lastres-Becker I, Brodesser S, Lütjohann D, Azizov M, Buchmann J, Hintermann $\mathrm{E}$, et al. Insulin receptor and lipid metabolism pathology in ataxin-2 knock-out mice. Hum Mol Genet. 2008;17:1465-1481.

9. Drost J, Nonis D, Eich F, Leske O, Damrath E, Brunt E, et al. Ataxin-2 modulates the levels of Grb2 and Src but not Ras signaling. J Mol Neurosci. 2013;51:68-81.

10. Auburger G, Gispert S, Lahut S, Omür O, Damrath E, Heck M, et al. 12 q24 locus association with type 1 diabetes: SH2B3 or ATXN2? World J Diabetes. 2014;5:316-327.

11. Flores-Alvarado LJ, Dávalos-Rodríguez NO, García-Cruz D, Madrigal-Ruiz PM, Ruiz-Mejía R, Aguilar-Aldrete ME, et al. (CAG)n polymorphism of the ATXN2 gene, a new marker of susceptibility for type 2 diabetes mellitus. Rev Panam Salud Publica. 2016;40:318-324.

12. Kraja AT, Chasman DI, North KE, Reiner AP, Yanek LR, Kilpeläinen TO, et al. Pleiotropic genes for metabolic syndrome and inflammation. Mol Genet Metab. 2014;112:317-338.
13. Zhang $H$, Mo XB, Xu T, Bu XQ, Lei SF, Zhang YH. Novel genes affecting blood pressure detected via gene-based association analysis. G3 (Bethesda). 2015;5:1035-1042.

14. Soranzo N, Spector TD, Mangino M, Kühnel B, Rendon A, Teumer A et al. A genome-wide meta-analysis identifies 22 loci associated with eight hematological parameters in the HaemGen consortium. Nat Genet. 2009;41:1182-1190.

15. Ikram MK, Sim X, Jensen RA, Cotch MF, Hewitt AW, Ikram MA, et al. Four novel Loci (19q13, 6q24, 12q24, and 5q14) influence the microcirculation in vivo. PLoS Genet. 2010;6:e1001184.

16. Ganesh SK, Zakai NA, Van-Rooij FJ, Soranzo N, Smith AV, Nalls MA, et al. Multiple loci influence erythrocyte phenotypes in the CHARGE Consortium. Nat Genet. 2009;41:1191-1198.

17. Meierhofer D, Halbach M, Şen NE, Gispert S, Auburger G. Ataxin-2 (Atxn2)-knock-out mice show branched chain amino acids and fatty. Mol Cell Proteomics. 2016;15:1728-1739.

18. Abdel-Aleem A, Zaki MS. Spinocerebellar ataxia type 2 (SCA2) in an Egyptian family presenting with polyphagia and marked CAG expansion in infancy. J Neurol. 2008;255:413-419.

19. Huynh DP, Maalouf M, Silva AJ, Schweizer FE, Pulst SM. Dissociated fear and spatial learning in mice with deficiency of ataxin-2. PLoS One. 2009;4:e6235.

20. Scoles DR, Meera P, Schneider MD, Paul S, Dansithong W, Figueroa KP, et al. Antisense oligonucleotide therapy for spinocerebellar ataxia type 2 . Nature. 2017;544:362-366

21. Li PP, Sun X, Xia G, Arbez N, Paul S, Zhu S, et al. ATXN2-AS, a gene antisense to $A T X N 2$, is associated with spinocerebellar ataxia type 2 and amyotrophic lateral sclerosis. Ann Neurol. 2016;80:600-615.

22. Wild PS, Felix JF, Schillert A, Teumer A, Chen MH, Leening MJG, et al. Large-scale genome-wide analysis identifies genetic variants associated with cardiac structure and function. J Clin Invest. 2017;127:1798-1812

23. Khurana V, Peng J, Chung CY, Auluck PK, Fanning S, Tardiff DF, et al. Genome-scale networks link neurodegenerative disease genes to $\alpha$-synuclein through specific molecular pathways. Cell Syst. 2017;4:157-170.

24. Almaguer-Mederos LE, Almaguer-Gotay $D$, Aguilera-Rodríguez $R$ González-Zaldívar Y, Cuello-Almarales D, Laffita-Mesa J, et al. Association of glutathione S-transferase omega polymorphism and spinocerebellar ataxia type 2. J Neurol Sci. 2017:372:324-328.

25. Ding D, Li K, Wang C, Chen Z, Long Z, Peng Y, et al. ATXN2 polymorphism modulates age atonsetin Machado-Joseph disease. Brain. 2016;139(10):e59.

26. Marthaler AG, Schmid B, Tubsuwan A, Poulsen UB, Engelbrecht AF, Mau-Holzmann UA, et al. Generation of an isogenic, gene-corrected control cell line of the spinocerebellar ataxia type 2 patient-derived iPSC line H271.Stem Cell Res. 2016;16:180-183.

27. Marthaler AG, Schmid B, Tubsuwan A, Poulsen UB, Hyttel P, Nielsen TT, et al. Generation of spinocerebellar ataxia type 2 patient-derived iPSC line H266. Stem Cell Res. 2016;16:166-169.

28. Marthaler AG, Tubsuwan A, Schmid B, Poulsen UB, Hyttel P, Nielsen JE, et al. Generation of spinocerebellar ataxia type 2 patient-derived iPSC line H271. Stem Cell Res. 2016;16:159-161.

29. Bailey JN, Loomis SJ, Kang JH, Allingham RR, Gharahkhani P, Khor CC, et al. Genome-wide association analysis identifies TXNRD2, ATXN2 and FOXC1 as susceptibility loci for primary open-angle glaucoma. Nat Genet. 2016;48:189-194.

30. Halbach MV, Gispert S, Stehning T, Damrath E, Walter M, Auburger G. Atxn2 knock-out and CAG42-knock-in cerebellum shows similarly dysregulated expression in calcium homeostasis pathway. Cerebellum. 2017;16:68-81. 